\title{
Clinical study of colorectal cancer operation: Survival analysis
}

\author{
Youngki Hong', Jaelim Kim¹, Yoon Jung Choi ${ }^{2}$, Jung Gu Kang ${ }^{1}$ \\ Departments of 'Surgery and ${ }^{2}$ Pathology, National Health Insurance Service Ilsan Hospital, Goyang, Korea
}

Purpose: Overall 5-year survival rates for colon and rectal cancer reported recently by the American Cancer Society were 89\%, 90\% for localized disease, 71\%, 71\% for regional disease, and 14\%, 15\% for distant disease. But the results of survival rate of colorectal cancer in a single institution were very rare. The aim of this study is to evaluate surgical results and survival rates of colorectal cancer.

Methods: We conducted a retrospective study with patients who underwent curative resection for a primary colorectal adenocarcinoma between 2009 and 2018.

Results: Five-year overall and disease-free survival were $79.5 \%$ and $69.9 \%$, respectively. The overall 5 -year survival by stages was $94.7 \%$ in stage I, $88.4 \%$ in stage II, $74.3 \%$ in stage III and $31.5 \%$ in stage IV. Five-year disease-free survival was $91 \%$ in stage I, $79.8 \%$ in stage II, $63.3 \%$ in stage III, and $18.9 \%$ in stage IV. The overall 5 -year survival in rectal cancer was superior to colon cancer $(P=0.014)$ while there was no difference in 5 -year disease-free survival $(P=0.338)$. Overall survival in female patients was better than male patient $(P=0.029)$. Overall survival by age was worst in group of less than 40 years old (62.5\%), and best in group between 41 and 65 years old (86.5\%). Postoperative mortality within 30 days was $0.4 \%$, and the recurrence rate was $19.2 \%$.

Conclusion: With the development of surgical skills and various treatments, postoperative outcome of colorectal cancer is expected to improve. It may be helpful for surgeons to improve their surgical outcomes when they review their data and conduct active researches.

Keywords: Colonic neoplasms, Rectal neoplasms, Survival rate

\section{INTRODUCTION}

Colorectal cancer is one of the most occurring malignancies, and the incidence is expected to increase in Korea with the aging population and westernized lifestyles [1]. Based on data from Korea Central Cancer Registry, the incidence rate of colorectal cancer increased from 20.7 (men, 22.5 and women, 18.9) per 1,000,000 in 1999 to 54.9 (men, 65.1 and women, 44.7) per 1,000,000 in 2017 [2]. Early diagnosis and proper management are the most import-

Received: Mar 20, 2020 Revised: Jun 2, 2020 Accepted: Jun 11, 2020 Correspondence to: Jung Gu Kang

Department of Surgery, National Health Insurance Service Ilsan Hospital, 100 Ilsan-ro, Ilsandong-gu, Goyang 10444, Korea

Tel: +82-31-900-0216, Fax: +82-31-900-0343

E-mail: kangski@nhimc.or.kr

ORCID: Youngki Hong (https://orcid.org/0000-0002-5767-9996), Jaelim Kim (https://orcid.org/0000-0001-8267-2748), Yoon Jung Choi (https://orcid.org/00000002-5701-8864), Jung Gu Kang (https://orcid.org/0000-0003-1282-5889)

Copyright $@ 2020$ Korean Society of Surgical Oncology

This is an Open Access article distributed under the terms of the Creative Commons Attribution Non-Commercial License (https://creativecommons.org/licenses/by-nc/4.0) which permits unrestricted non-commercial use, distribution, and reproduction in any medium, provided the original work is properly cited. ant in the treatment of colon cancer. When it is resectable, colon cancer can be managed with surgery only, or surgery with adjuvant chemotherapy whereas rectal cancer requires surgery only in early stage or surgery with neoadjuvant or adjuvant chemoradiation in advanced stage. In addition, the survival rate for colorectal cancer have been improved due to the recent increase of early diagnosis, improvement in surgery, development of chemotherapy agent, and improvement of neoadjuvant chemoradiation treatments. The 5-year survival rate of colorectal cancer in Korea was also improved from $54.8 \%$ in 1995 to $74.8 \%$ in 2012 [1]. However, there have been few recent reports on the survival rate of colorectal cancer in Korea.

The aim of this study was to evaluate the clinical characteristics and oncologic outcomes of colorectal cancer patients who underwent curative surgery followed with or without adjuvant chemotherapies.

\section{METHODS}

Patients

We reviewed the medical records of patients who underwent radi- 
cal surgery for pathologically proven primary colorectal adenocarcinoma between 2009 and 2018 in National Health Insurance Service Ilsan Hospital, Goyang, Korea. Patients who underwent palliative resection or bypass surgery were excluded from this study. Patients with Tis (carcinoma in situ) adenocarcinoma and other colorectal cancer except adenocarcinoma such as lymphoma, sarcoma, and gastrointestinal stromal tumor were also excluded. This study was reviewed and approved by the institutional review board (IRB No. NHIMC 2018-09-010). The medical records of the patients were collected and reviewed retrospectively to evaluate the patients' characteristics, operative data and survival outcomes.
Colorectal cancer was divided into colon cancer and rectal cancer, and the rectosigmoid colon cancer was classified into colon cancer. Colon cancer can be subdivided to appendiceal cancer, cecal cancer, ascending colon cancer, hepatic flexure cancer, transverse colon cancer, splenic flexure cancer, descending colon cancer, sigmoid colon cancer, and rectosigmoid colon cancer according to the anatomic location. Colon cancer located from appendix to transverse colon was categorized as right-side colon cancer and colon cancer from splenic flexure colon to rectosigmoid colon was categorized as left-side colon cancer in this study.

The analysis included sex, age, name of operation, date of opera-

Table 1. Patients' characteristics

\begin{tabular}{|c|c|c|c|c|}
\hline Characteristics & Total & Colon & Rectal & P-value \\
\hline Sex & & & & 0.125 \\
\hline Male & $683(60.7)$ & $440(59.1)$ & $243(63.8)$ & \\
\hline Female & $443(39.3)$ & $305(40.9)$ & $138(36.2)$ & \\
\hline Age (yr) & $67.4 \pm 11.9$ & $68.3 \pm 11.9$ & $65.7 \pm 11.7$ & 0.001 \\
\hline$\leq 40$ & $18(1.6)$ & $10(1.3)$ & $8(2.1)$ & 0.006 \\
\hline $41-64$ & $404(35.9)$ & $245(32.9)$ & $159(41.7)$ & \\
\hline$\geq 65$ & $704(62.5)$ & $490(65.8)$ & $214(56.2)$ & \\
\hline Stage & & & & $<0.001$ \\
\hline 1 & $241(21.4)$ & 118 (15.8) & $123(32.3)$ & \\
\hline$\|$ & $382(33.9)$ & $265(35.6)$ & $117(30.7)$ & \\
\hline III & $392(34.8)$ & $284(38.1)$ & $108(28.3)$ & \\
\hline IV & $111(9.9)$ & 78 (10.5) & $33(8.7)$ & \\
\hline Total & $1,126(100)$ & 745 (66.2) & $381(33.8)$ & \\
\hline Preoperative CEA level $(n=1,016)$ & & & & 0.166 \\
\hline$<5$ & 740 (72.8) & $475(71.4)$ & $265(75.5)$ & \\
\hline$\geq 5$ & $276(27.2)$ & $190(28.6)$ & $86(24.5)$ & \\
\hline Total & $1,016(100)$ & $665(65.5)$ & $351(34.5)$ & \\
\hline Associated polyp $(n=1,063)$ & & & & 0.025 \\
\hline \multicolumn{5}{|l|}{ (No. of polyps) } \\
\hline 0 & $628(55.8)$ & 393 (52.8) & $235(61.7)$ & \\
\hline 1 & $190(16.9)$ & $133(17.9)$ & $57(15.0)$ & \\
\hline 2 & $113(10.0)$ & $83(11.1)$ & $30(7.9)$ & \\
\hline 3 & $71(6.3)$ & $56(7.5)$ & $15(3.9)$ & \\
\hline 4 & $47(4.2)$ & $31(4.2)$ & $16(4.2)$ & \\
\hline$\geq 5$ & $77(6.8)$ & $49(6.6)$ & $28(7.3)$ & \\
\hline Total & $1,126(100)$ & 745 (66.2) & 381 (33.8) & \\
\hline Differentiation $(n=1,047)$ & & & & 0.008 \\
\hline Well & $279(26.6)$ & $180(25.9)$ & $99(28.0)$ & \\
\hline Moderate & $688(65.7)$ & $447(64.4)$ & $241(68.3)$ & \\
\hline Poorly & $59(5.6)$ & $50(7.2)$ & $9(2.5)$ & \\
\hline Mucinous & $21(2.0)$ & $17(2.4)$ & $4(1.1)$ & \\
\hline Total & $1,047(100)$ & $694(66.3)$ & 353 (33.7) & \\
\hline
\end{tabular}

Values are presented as number (\%) or mean \pm standard deviation. CEA, carcinoembryonic antigen. 
tion, pathologic stage, preoperative carcinoembryonic antigen (CEA) levels, associated polyp, tumor differentiation, recurrence and survival. Tumor stages were determined according to the American Joint Committee on Cancer (AJCC) staging, 8th edition. Postoperative death was defined as death within 30 days from surgery.

\section{Statistical analyses}

All statistical analyses were performed using SAS statistical software version 9.3 (SAS Institute Inc., Cary, NC, USA). The results were presented as means with standard deviations (mean $\pm \mathrm{SD}$ ) for continuous outcomes, and as frequencies and percentages for categorical outcomes. Categorical variables were compared using chisquare or Fisher exact tests, and continuous variables were compared using independent sample t-tests. The survival rate was estimated by the Kaplan-Meier method and compared by the logrank test. Multivariate analyses were performed by using Cox's proportional hazards model to identify prognostic factors. P-value of less than 0.05 was considered statistically significant.

\section{RESULTS}

Between 2009 and 2018, 1,126 patients underwent radical surgery for colorectal adenocarcinoma, and Table 1 summarizes patients' characteristics. Of these patients, 683 patients $(60.7 \%)$ were males, and there were 18 patients under the age of $40(1.6 \%), 404$ patients of the age between 41 and 65 years old (35.9\%), and 704 patients $(62.5 \%)$ aged 65 years old or older. By stage, there were 241 patients (21.4\%) in stage I, 382 patients (33.9\%) in stage II, 392 patients (34.8\%) in stage III, and 111 patients $(9.9 \%)$ in stage IV. The preop-

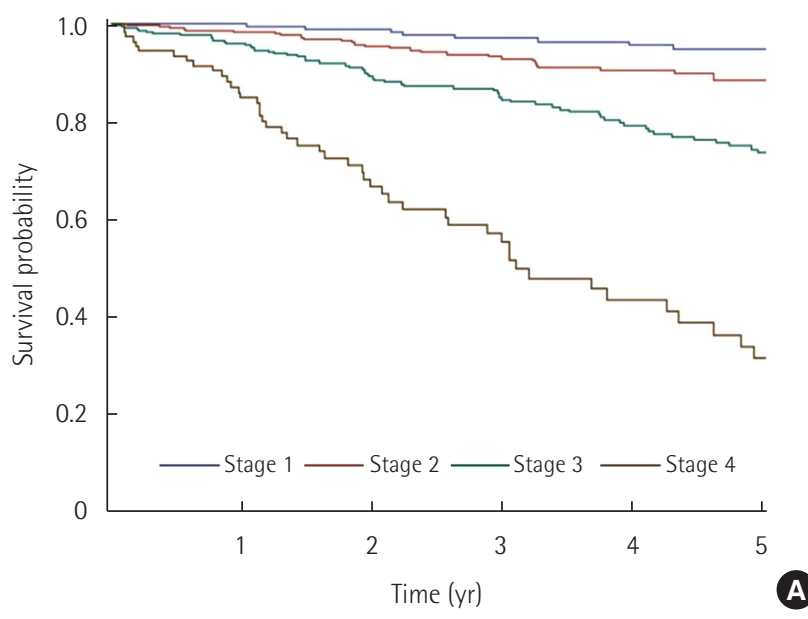

erative CEA level was more than $5 \mathrm{ng} / \mathrm{dL}$ in 276 patients (27.2\%). The preoperative colonoscopy identified associated polyps in $44.2 \%$ of patients (colon cancer, $48.2 \%$ and rectal cancer, $38.2 \%$ ). According to tumor differentiation, moderate differentiation type was the most common with 688 patients (65.7\%). According to the anatomic location, right-side colon cancer, left-side colon cancer, and rectal cancer were found in 307 patients (27.2\%), 441 patients (39.2\%), and 378 patients (33.6\%), respectively. Of these patients, four patients died within 30 days from surgery (mortality rate, $0.4 \%$ ), and the causes of death were two respiratory failures, one cardiac shock, and one liver failure who underwent neoadjuvant chemoradiation for rectal cancer.

The median follow-up duration was 36.3 months (range, 0.1120.3 months). During the follow-up period, 158 patients (14.0\%) out of 1,126 patients died. These 158 patients included 114 colon cancer patients out of 745 patients (15.3\%), and 44 rectal cancer patients out of 381 patients (11.5\%). The recurrence occurred in 216 patients out of 1,126 patients (19.2\%) including 136 patients (18.2\%) with colon cancer, and 80 patients (21.0\%) with rectal cancer. Of these recurrences in rectal cancer, local recurrence accounted for nine patients (2.4\%), and there was no local recurrence in patients with colon cancer. Overall 5-year survival was 79.5\%, and 5-year disease-free survival was 69.9\%. Overall 5-year survival was significantly higher in rectal cancer than colon cancer $(84.2 \%$ vs. $76.9 \%, \mathrm{P}=0.014$ ), whereas there was no significant difference in 5 -year disease-free survival between colon cancer and rectal cancer (69.8\% vs. $70.2 \%, \mathrm{P}=0.338)$. Overall 5 -year survival rates and 5-year disease-free survival rates according to stage were $94.7 \%$ and $91.0 \%$ in stage I, $88.4 \%$ and $79.8 \%$ in stage II, $74.3 \%$ and $63.3 \%$ in stage III, and $31.5 \%$ and $18.9 \%$ in stage IV (Fig. 1). Overall sur-

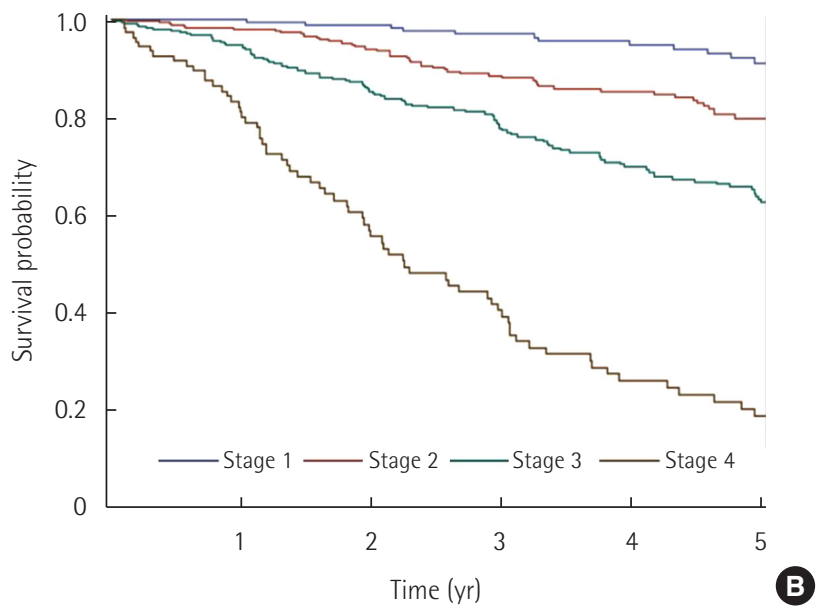

Fig. 1. Survival analysis according to stages. (A) Overall 5-year survival rate: $94.7 \%$ in stage I, 88.4\% in stage II, $74.3 \%$ in stage III, 31.5\% in stage IV $(\mathrm{P}<0.001)$. (B) Five-year disease-free survival rate: $91.0 \%$ in stage I, 79.8\% in stage II, 63.3\% in stage III, 18.9\% in stage IV $(\mathrm{P}<0.001)$. 
Table 2. Multivariate analysis of clinicopathological factors for overall survival

\begin{tabular}{lrc}
\hline Variable & P-value & HR (95\% Cl) \\
\hline Colon cancer & 0.134 & $1.347(0.912-1.989)$ \\
Male sex & 0.003 & $1.876(1.247-2.823)$ \\
Age $\leq 40$ years & 0.569 & $1.508(0.367-6.188)$ \\
Preoperative CEA $\geq 5 \mathrm{ng} / \mathrm{dL}$ & $<0.001$ & $2.217(1.544-3.184)$ \\
Poor/mucinous differentiation & 0.030 & $1.957(1.069-3.585)$ \\
Lymphovascular invasion & 0.059 & $1.440(0.986-2.103)$ \\
\hline
\end{tabular}

$\mathrm{HR}$, hazard ratio; $\mathrm{Cl}$, confidence interval; $\mathrm{CEA}$, carcinoembryonic antigen.

vival and disease-free survival were significantly higher in female patients than male patients $(\mathrm{P}=0.029$ and $\mathrm{P}=0.041$, respectively). The overall 5-year survival $(\mathrm{P}=0.001)$ and 5 -year disease-free survival $(\mathrm{P}=0.014)$ were lowest in patient under 40 years old with $62.5 \%$ and $52.1 \%$ whereas highest in patients aged 41 to 65 years old with $86.5 \%$ and $74.9 \%$. There was significant difference in overall 5-year survival and 5-year disease-free survival rate according to preoperative CEA level $(\mathrm{P}<0.001$, both). Overall 5-year survival and 5-year disease-free survival rate in patients with poorly differentiated type were $54.9 \%$ and $32.7 \%$, and there were statistical significances $(\mathrm{P}<0.001$, both). Multivariate analysis revealed that male sex, elevated preoperative CEA level, and poorly or mucinous differentiation were poor prognostic factors for overall survival, whereas elevated preoperative CEA level, poorly or mucinous differentiation and presence of lymphovascular invasion were poor prognostic factors for disease-free survival (Tables 2, 3).

\section{DISCUSSION}

Various kinds of treatments have been developed with advance in basic research, clinical study, and research of carcinogenesis for colorectal cancer and their effectiveness has been reported in various ways [3-6]. Efforts to predict the prognosis and recurrence of colorectal cancer have been developed, but to date, the most powerful predictor of patients' prognosis is TNM stage. The current stage of colorectal cancer is a combination of depth of invasion (T), lymph node metastasis $(\mathrm{N})$, and distant metastasis $(\mathrm{M})$ by AJCC classification system, which was also used in this study [7].

The incidence of colorectal cancer in Korea has increased remarkably during the past few decades [2]. Accordingly, the treatment of colorectal cancer, especially in advanced and metastatic cancer, has advanced and the result of treatment has improved. The survival rate by the AJCC system has been reported more than $90 \%$ in stage I, $70 \%$ to $85 \%$ in stage II, $25 \%$ to $80 \%$ in stage III, and less than $10 \%$ in stage IV $[8,9]$. In this study, overall 5 -year survival rate was $79.5 \%$, and 5-year disease-free survival rate was $69.9 \%$.
Table 3. Multivariate analysis of clinicopathological factors for disease-free survival

\begin{tabular}{lcc}
\hline Variable & P-value & HR $(95 \% \mathrm{Cl})$ \\
\hline Colon cancer & 0.110 & $0.781(0.577-1.057)$ \\
Male sex & 0.393 & $1.144(0.840-1.557)$ \\
Age $\leq 40$ years & 0.966 & $1.025(0.325-3.230)$ \\
Preoperative CEA $\geq 5 \mathrm{ng} / \mathrm{dL}$ & $<0.001$ & $2.380(1.770-3.201)$ \\
Poor/mucinous differentiation & 0.003 & $2.031(1.266-3.258)$ \\
Lymphovascular invasion & $<0.001$ & $2.116(1.531-2.925)$ \\
\hline
\end{tabular}

$H R$, hazard ratio; $\mathrm{Cl}$, confidence interval; $C E A$, carcinoembryonic antigen.

The overall 5-year survival rate by stage was $94.7 \%$ in stage I, $88.4 \%$ in stage II, $74.3 \%$ in stage III, and $31.5 \%$ in stage IV, which is more favorable compared to previous literatures, 5-year disease-free survival was $91.0 \%$ in stage I, $79.8 \%$ in stage II, 63.3 in stage III, and $18.9 \%$ in stage IV. Jung et al. [1] reported frequency, mortality, and survival rate of colorectal cancer based on data of the Korea $\mathrm{Na}$ tional Cancer Incidence Database in 2015. Colorectal cancer was the fourth most common cause of death in men and second in women. They reported that the survival rate for patients with colorectal cancer between 2008 and 2012 was 74.8\% (76.9\% in male patients and $71.8 \%$ in female patients) and the survival rate has improved compared to the past periods. The survival rate of $79.5 \%$ ( $76.4 \%$ in male patients and $84.7 \%$ in female patients) may be regarded as improving results over time.

Lee et al. [10] reported the survival rate in 689 patients with colorectal cancer according to subside of colon and rectum, which showed overall 5 -year survival rate of $77.8 \%$ including $92.9 \%$ in cecal cancer, $69.5 \%$ in ascending colon cancer, $76.2 \%$ in transverse colon cancer, $84.0 \%$ in descending colon cancer, $82.2 \%$ in sigmoid colon cancer, and $77.5 \%$ in rectal cancer. In the United States, about 145,600 patients are diagnosed as colorectal cancer per year including 101,420 patients with colon cancer and 44,180 patients with rectal cancer. Overall 5-year survival rates for these patients were $65 \%$, including $90 \%$ for localized disease, $71 \%$ for regional disease, and 14\% for distant disease. In addition, overall 5-year survival rates for colon cancer was $64 \%$, including $90 \%$ for localized disease, $71 \%$ for regional disease, and $14 \%$ for distant disease while overall 5 -year survival rates for rectal cancer was $67 \%$, including $89 \%$ for localized disease, $71 \%$ for regional disease, and $15 \%$ for distant disease [11]. These results are similar to the data between 2009 and 2015 which showed overall 5-year survival rates of 63\% for colon cancer, including 90\% for localized disease, $71 \%$ for regional disease, and $14 \%$ for distant disease while overall 5-year survival rates of $67 \%$ for rectal cancer, including $89 \%$ for localized disease, $71 \%$ for regional disease, and 15\% for distant disease [12]. However, the overall 5-year survival and 5-year disease-free sur- 
vival for distant disease in our study were $31.5 \%$ and $18.9 \%$, respectively. Recent aggressive surgical treatment and advanced chemotherapy regimen for metastatic colorectal cancer seem to have improved the survival rate.

As an aging society progresses, there is a growing interest in colorectal cancer surgery in elderly patients. According to Seow-En et al. [13] who reported surgical outcomes in patients with advanced age, median overall survival was 78 months. Following multivariate analysis, the factors significant for predicting overall mortality were serum albumin $<35 \mathrm{~g} / \mathrm{dL}$, serum CEA $\geq 20 \mu \mathrm{g} / \mathrm{L}$, T stage 3 or 4 , moderate tumor cell differentiation or worse, mucinous histology, rectal tumors, and preexisting chronic obstructive lung disease. However, advanced age alone was not found to be significant. In our study, we divided the age group into 40 years old and younger, 41 to 64 years old and 65 years old or older. The age group of 40 years old and younger had the lowest overall 5-year survival rate of $6.25 \%$ and overall 5 -year survival of $75.7 \%$ in 65 years old and older group was similar to previous literatures.

Various factors such as tumor stage, preoperative CEA, cell differentiation, vascular invasion, intestinal obstruction, intestinal perforation, age, and sex have been reported as predictive factors on survival rate following curative resection for colorectal cancer [14-16]. In our study, there was significant difference in survival rate according to tumor stage, preoperative CEA, age, and sex. Recurrence rate after curative resection for colorectal cancer has been reported as 30\% to 50\% [17]. Ryuk et al. [16] defined early recurrence as recurrence within 2 years after curative resection. The liver was the most common site of early recurrence $(40.5 \%)$, whereas late recurrence was more common locally (28.1\%), or in the lung (32.8\%). The 5 -year overall survival rates for early and late recurrence were $34.7 \%$ and $78.8 \%$, respectively. The recurrence rate in our study was $19.2 \%$.

Tekkis et al. [18] reported the 30-day mortality of 7.5\% in 8,077 patients with colorectal cancer. They suggested age, American Society of Anesthesiology grade, Dukes' stage, and emergency operation as independent risk factors for mortality. Rutegard et al. [19] reported that mortality within 30 days of surgery decreased from $2.1 \%$ in $2000-2003$ to $1.6 \%$ in $2008-2011$. Although the mortality rate varies by reporters, postoperative mortality is still a major issue of concern. To improve the survival rates, low mortality rate could also be a point to consider. With improvement of surgical skills as well as medical supports, the mortality rate is expected to become lower. The postoperative 30 -day mortality in our study was $0.4 \%$. Although we did not analyze the risk factors for the 30-day mortality because of the low rate of mortality, surgeons should pay a great attention to avoid the mortality in the perioperative period.

With the development of surgical skills and various treatments, postoperative outcome of colorectal cancer is expected to improve. It may be helpful for surgeons to improve their surgical outcomes when they review their data and conduct active researches.

\section{CONFLICT OF INTEREST}

No potential conflict of interest relevant to this article was reported.

\section{ACKNOWLEDGMENTS}

We would like to thank Jung-Kyu Choi in research support team for preparing the tables and figures in this study.

\section{REFERENCES}

1. Jung KW, Won YJ, Kong HJ, Oh CM, Cho H, Lee DH, et al. Cancer statistics in Korea: incidence, mortality, survival, and prevalence in 2012. Cancer Res Treat 2015;47:127-41.

2. Korea Central Cancer Registry. Statics (2007-2017) [Internet]. Goyang (KR): Korea Central Cancer Registry; c2020 [cited 2020 Jan 29]. Available from: https://kccrsurvey.cancer.go.kr:10443/ sub01/sub01_03.jsp.

3. Fan S, Li T, Zhou P, Peng Q, Zhu Y. Development and validation of nomogram combining serum biomarker for predicting survival in patients with resected rectal cancer. Biosci Rep 2019;39:BSR 20192636.

4. Kim S, Ko D, Lee Y, Jang S, Lee Y, Lee IY, et al. Anti-cancer activity of the novel 2-hydroxydiarylamide derivatives IMD-0354 and KRT1853 through suppression of cancer cell invasion, proliferation, and survival mediated by TMPRSS4. Sci Rep 2019;9:10003.

5. Xu H, You G, Zhang M, Song T, Zhang H, Yang J, et al. Association of pre-surgery to pre-radiotherapy lymphocyte counts ratio with disease-free survival in rectal cancer patients receiving neoadjuvant concurrent chemoradiotherapy. World J Surg Oncol 2019;17:199.

6. Kim JH, Kim CN, Kang DW. Squalene epoxidase correlates e-cadherin expression and overall survival in colorectal cancer patients: the impact on prognosis and correlation to clinicopathologic features. JClin Med 2019;8:632.

7. Amin MB, Edge S, Greene F, Byrd DR, Brookland RK, Washington MK, et al. AJCC cancer staging manual. 8th ed. New York, NY: Springer; 2018.

8. O'Connell JB, Maggard MA, Ko CY. Colon cancer survival rates with the new American Joint Committee on Cancer sixth edition staging. J Natl Cancer Inst 2004;96:1420-5.

9. Meyerhardt JA, Mayer RJ. Systemic therapy for colorectal cancer. N Engl J Med 2005;352:476-87.

10. Lee I, Baek SH, Kim H, Jo HJ, Oh NG, Ko S. Survival analysis for 
colon subsite and rectal cancers: experience from a single surgeon. Korean J Clin Oncol 2015;11:114-9.

11. American Society of Clinical Oncology. Colorectal cancer: statistics [Internet]. Alexandria (VA): American Society of Clinical Oncology; c2018 [cited 2019 Dec 10]. Available from: https://www.cancer. net/cancer-types/colorectal-cancer/statistics.

12. American Cancer Society. Survival rates for colorectal cancer [Internet]. Atlanta (GA): American Cancer Society; c2019 [cited 2019 Dec10]. Available from: https:/www.cancer.org/cancer/colon-rectal-cancer/detection-diagnosis-staging/survival-rates.html.

13. Seow-En I, Tan WJ, Dorajoo SR, Soh SHL, Law YC, Park SY, et al. Prediction of overall survival following colorectal cancer surgery in elderly patients. World J Gastrointest Surg 2019;11:247-60.

14. Wanebo HJ, Rao B, Pinsky CM, Hoffman RG, Stearns M, Schwartz $\mathrm{MK}$, et al. Preoperative carcinoembryonic antigen level as a prognostic indicator in colorectal cancer. N Engl J Med 1978;299:44851.
15. Abdalmassih M, Sivananthan G, Raizman Z, Lambert P, Wirtzfeld D, Bashir B, et al. Prognostic markers of recurrence and survival in rectal cancer treated with neoadjuvant chemoradiotherapy and surgery. Colorectal Cancer 2018;7:CRC02.

16. Ryuk JP, Choi GS, Park JS, Kim HJ, Park SY, Yoon GS, et al. Predictive factors and the prognosis of recurrence of colorectal cancer within 2 years after curative resection. Ann Surg Treat Res 2014;86: 143-51.

17. Abulafi AM, Williams NS. Local recurrence of colorectal cancer: the problem, mechanisms, management and adjuvant therapy. Br J Surg 1994;81:7-19.

18. Tekkis PP, Poloniecki JD, Thompson MR, Stamatakis JD. Operative mortality in colorectal cancer: prospective national study. BMJ 2003;327:1196-201.

19. Rutegard M, Haapamaki M, Matthiessen P, Rutegard J. Early postoperative mortality after surgery for rectal cancer in Sweden, 20002011. Colorectal Dis 2014;16:426-32. 